\title{
Prevalencia de bacterias causantes de mastitis en fincas lecheras de Toca (Boyacá, Colombia)
}

\author{
Prevalence of Mastitis Causing - Bacteria in the \\ Milking Farms of Toca (Boyacá, Colombia)
}

Fecha recepción: 13 de octubre de 2013 Fecha Aprobación: 12 de enero de 2014
Roy José Andrade-Becerra ${ }^{2}$ Zonia Elizabeth Caro-Carvajal'2, Martín Orlando Pulido-Medellín ${ }^{3}$, José Luis Porras-Vargas ${ }^{4}$, Julio César Vargas-Abella ${ }^{5}$

\section{RESUMEN}

El presente trabajo tuvo como objetivo aislar las diferentes bacterias causantes de mastitis subclínica, de cuartos positivos a CMT $(2+)$ o mayores a $300,000 \mathrm{cel} / \mathrm{mL}$ de vacas procedentes de fincas lecheras del municipio de Toca (Boyacá). Mediante un muestreo probabilístico de conveniencia y por medio de un estudio descriptivo longitudinal retrospectivo durante el año 2013, se estudiaron 130 fincas especializadas en la producción de leche con promedios de 20 animales en lactancia por finca, ubicadas entre los 2300 y los 2800 msnm, con temperaturas anuales promedios de $13{ }^{\circ} \mathrm{C}$ y pluviosidades entre 800 y 1200 mm/año. Durante el año de estudio se encontró un $3.14 \%$ de cuartos positivos al CMT con grados superiores a $2+$. Streptococcus agalactiae se aisló en el 9,73\%, y se convirtió en el principal patógeno aislado. Staphylococcus aureus fue aislado, en promedio, en el $6,00 \%$.

\begin{abstract}
This paper had as objective the isolation of different bacteria, which causes mastitis, and simultaneously, to make an emphasis on the differentiation of Mycoplasma species, taken from stored milk samples and from every cow individually. These cows and the milk were provided by farms from the Toca in Boyacá. This process was carried out through a convenience probabilistic sampling and also a descriptive, longitudinal and retrospective research during 2013. The research took into account 130 technicalizated milking farms, where every one has approximately 20 milking cows. These farms are located between 2300 and 2800 meters above sea level and have temperatures among $55^{\circ} \mathrm{F}$ and a rain range of 800 and $1200 \mathrm{~mm} \mathrm{Hg}$ per year. During the study it was found a $3.14 \%$ positive to CMT in udder quarters with grades greater than 2 + . For this purpose the Streptococcus agalactiae
\end{abstract}

Ph.D. Universidad Pedagógica y Tecnológica de Colombia (Tunja, Boyacá-Colombia). Correo electrónico: royandrade@ uptc.edu.co

2 Profesional Independiente. Correo electrónico: zelizabeth-92@hotmail.com.

3 M.Sc. Universidad Pedagógica y Tecnológica de Colombia (Tunja, Boyacá-Colombia). Correo electrónico: martin.pulido@ uptc.edu.co

$4 \quad$ M.Sc. Universidad Pedagógica y Tecnológica de Colombia (Tunja, Boyacá-Colombia). Correo electrónico: jose.porras@ uptc.edu.co

5 Universidad Pedagógica y Tecnológica de Colombia (Tunja, Boyacá-Colombia). Correo electrónico: julio.vargas@uptc.edu.co 
Roy José Andrade-Becerra, Zonia Elizabeth Caro-Carvajal, Martín Orlando Pulido-Medellín,

José Luis Porras-Vargas, Julio César Vargas-Abella

Corynebacterium bovis se aisló en el 0,30\% de los casos. E. coli no se aisló. Mycoplasma bovis no se encontró en ninguna muestra. $M$. californicum se recuperó en el 0,50\%. Levaduras, en el 0,20\%, y Acholeplasma spp. se aisló en el $0,40 \%$.

Palabras clave: Mastitis Subclínica, Leche, Vacas. was isolated at $9.73 \%$ and it turned to be the most isoleted pathogen. (Staphylococcus aureus was isolated about $6 \%$ average Corynebacterium bovis was isolated in the $0,30 \%$ of the cases. E. coli was not isolated. Also the sampling didn't find any Mycoplasma bovis in three years. $M$ californicum was recovered in the $0.50 \%$. Leavens in the $20 \%$ and Acholeplasma spp. was isoleted in the $0.40 \%$.

Key Words: Prevalence, Subclinical Mastitis, Milk, Cattle. 


\section{INTRODUCCIÓN}

La mastitis es una inflamación de la glándula mamaria causada, en la mayoría de los casos, por acción bacteriana; su prevalencia varía ampliamente entre zonas y entre fincas, y cambia de un momento a otro en una misma finca $(1,2)$. Las bacterias que la causan varían y reflejan los factores predisponentes involucrados (3), pero se han señalado los patógenos Streptococccus agalactiae y Staphylococcus aureus como responsables del 80 al $90 \%$ de los casos (4). Esta enfermedad es considerada la más costosa en las explotaciones lecheras del mundo; a la forma subclínica de ella se le atribuye la mayor parte de las pérdidas $(5,6)$, por ser mucho más frecuente que la clínica (7) y por ser la causa más importante de las alteraciones que comprometen la calidad de la leche.

La producción de leche en el altiplano Boyacense se ha incrementado durante los últimos diez años a una tasa del $10 \%$ anual, aproximadamente, alcanzando un promedio de producción de 1.500.000 de litros de leche al día, entregada a las industrias lácteas en 2012 (8). Más del 90\% de las fincas lecheras ordeñan de forma manual, y como medida de control de mastitis, los productores desinfectan los pezones después del ordeño, en forma rutinaria.

En el altiplano Boyacense se ha observado en los últimos años que algunas de las grandes fincas lecheras han estado presentando alta incidencia de mastitis subclínica, superior al $40 \%$, la cual no responde a los tratamientos convencionales con antibióticos (8), y presenta altos recuentos de células somáticas en leche y caída abrupta del volumen, y los resultados son negativos en los medios de cultivo convencionales. Sin embargo, ningún intento se ha realizado para aislar otros patógenos de mastitis diferentes a los tradicionales.

El presente artículo informa sobre el aislamiento de las diferentes bacterias causantes de mastitis subclínica de cuartos positivos a CMT $(2+)$ o mayores a $300,000 \mathrm{cel} / \mathrm{mL}$ de vacas procedentes de fincas lecheras del altiplano Boyacense durante el año 2013.

\section{MATERIALES Y MÉTODOS}

Tipo de estudio. Mediante un muestreo probabilístico de conveniencia y por medio de un estudio descriptivo longitudinal retrospectivo (12), durante el año 2013 se estudiaron 130 fincas especializadas en la producción de leche, con promedios de 20 animales en lactancia por finca, ubicadas entre los 2300 y los 2800 msnm, con temperaturas anuales promedios de $13{ }^{\circ} \mathrm{C}$ y pluviosidades entre 800 y 1200 mm/año (13). Todas las fincas realizaban dos ordeños manuales por día. La base genética del ganado era $100 \%$ Holstein Friesian y el principal recurso forrajero fue el pasto kikuyo (Pennisetum clandestinum).

Se tomaron 863 vacas de leche, 3452 cuartos en total. La producción de leche promedio estaba en 4,500 L/vaca/año, y el número de células somáticas por cuarto fue, en promedio, de 300,000 cel/mL.

Exámenes bacteriológicos. De enero a diciembre de 2013 se ejecutaron pruebas de California Mastitis Test (CMT). De los cuartos resistentes a los tratamientos con antibióticos y de los cuartos positivos (CMT mayor o igual a $2+$ ) se tomaron muestras de leche en frascos estériles, previa limpieza y desinfección de la punta del pezón; se siguió el procedimiento descrito por National Mastitis Council (14). Lavado de pezones, secado y desinfección con solución jabonosa, luego se enjuagó con agua estéril, se secó con gasa estéril y se desinfectó con solución iodada; una vez finalizada la asepsia se descartó el primer chorro de leche, y se recolectó en bolsas de plástico nuevas y estériles (Whirl-pak). Las muestras recolectadas fueron mantenidas y transportadas en cavas refrigeradas hasta su llegada al laboratorio para su análisis.

Las muestras fueron tomadas por el veterinario y transportadas al Laboratorio de Microbiología de la Universidad Pedagógica y Tecnológica de Colombia en Tunja. El Laboratorio de Microbiología procede con el diagnóstico rutinario, así como con el aislamiento de gérmenes no comunes, siguiendo la metodología propuesta en el Laboratory Handbook on Bovine Mastitis, del Consejo Nacional de la Mastitis de los EE. UU. (14). 
Roy José Andrade-Becerra, Zonia Elizabeth Caro-Carvajal, Martín Orlando Pulido-Medellín,

José Luis Porras-Vargas, Julio César Vargas-Abella

Tipificación bacteriana. Para el aislamiento, las muestras se dejaron a temperatura ambiente hasta que alcanzaron los $19^{\circ} \mathrm{C}$; se tomaron alícuotas de $25 \mu$ l de cada muestra de leche, usando asa de platino calibrada, y se sembraron por agotamiento en agar base sangre (sangre de ovino para determinar tipo de hemólisis, y agar MacConkey para identificar enterobacterias y diferenciar tipo de fermentación), y se incubaron por 24 a 48 horas. Luego del crecimiento se realizó coloración de Gram, para clasificar las bacterias en Grampositivas y Gram-negativas, y, al mismo tiempo, según su morfología, en cocos o bacilos. A los cocos Gram-positivos se les realizó la prueba de catalasa, y se diferenciaron como coagulasas positivos y negativos. Luego se llevó la colonia a un sistema comercial ID32 STAPH, para identificar y confirmar género y especie; así se diferenció el Staphylococcus aureus de otros Staphylococcus coagulasa positivo. A los Streptococcus se les efectuó CAMP, hidrólisis de esculina, hipurato e inulina y crecimiento en $\mathrm{NaCl}$. A los bacilos Gram negativos se les sometió a las pruebas de oxidasa, indol, triple azúcar, RM-VP, LIA, urea y citrato.

Para Mycoplasma de las muestras de leche se sembró $0.01 \mathrm{~mL}$ de cada muestra individual en placas con medio solido de Hayflick modificado (15) mediante el empleo de asa de platino; las placas se incubaron a $37{ }^{\circ} \mathrm{C}$ en cámara húmeda con $10 \%$ de $\mathrm{CO}_{2}$ durante 10 días; luego de aislados se sometieron a sensibilidad a la digitonina para la diferenciación del genero Acholeplasma (16), fermentación de la glucosa (17), hidrólisis de la arginina (16), reducción del tetrazolium (17) y detección de la producción de film \& spot (17). El total de cuartos de animales con mastitis clínica se observa en la (Tabla I).

Análisis estadístico. Los datos obtenidos se registraron en la base de datos con la ayuda del ordenador dBASE 5.0., y fueron analizados estadísticamente mediante el programa SPSS para Windows versión 17. Se utilizó estadística descriptiva (12). Los resultados del análisis estadístico fueron interpretados con un nivel de confianza del $95 \%$.

\section{RESULTADOS Y DISCUSIÓN}

La inspección de la totalidad de los animales en cada finca permitió establecer que durante el año
2013 se encontraron, en promedio, el 5.65\% de cuartos de animales con mastitis clínica, los cuales no fueron incluidos en este estudio. Para determinar el daño ocasionado en el cuarto se tuvo en cuenta el CMT, el conteo de células somáticas y la palpación del respectivo cuarto durante el año de estudio, tal como se muestra en la Tabla I.

Tabla I. Total de cuartos de animales con mastitis clínica

\begin{tabular}{|l|c|l|}
\hline \multicolumn{1}{|c|}{ Resultado } & \multicolumn{2}{|c|}{$\begin{array}{l}\text { 2013 } \\
\text { n:10400 cuartos } \\
\text { examinados }\end{array}$} \\
\hline Cuartos perdidos & 1 & $0.83 \%$ \\
\hline Cuartos atrofiados & 8 & $6.66 \%$ \\
\hline $\begin{array}{l}\text { Daños severos con aumento de } \\
\text { volumen }\end{array}$ & 4 & $3.33 \%$ \\
\hline Daño severo sin aumento de volumen & 1 & $0.83 \%$ \\
\hline $\begin{array}{l}\text { Cambio con presencia de grandes } \\
\text { nódulos }\end{array}$ & 1 & $0.83 \%$ \\
\hline $\begin{array}{l}\text { Cambio con presencia de pequeños } \\
\text { nódulos }\end{array}$ & 12 & $10 \%$ \\
\hline Hiperqueratosis & 2 & $1.16 \%$ \\
\hline Eczema & 1 & $0.83 \%$ \\
\hline Cuartos con mastitis clínica & 30 & $25 \%$ \\
\hline Cuartos sin cambios clínicos & 10340 & $89.65 \%$ \\
\hline
\end{tabular}

De un total de cuartos negativos a mastitis clínica resultaron un total en el año 2013 de 10340 cuartos resultaron $470(4.54 \%)$ positivos al CMT con grados superiores a $2+$, resultados que difieren de los obtenidos por Alonso et al. (2010), quienes sobre un total de 6.975 cuartos reportan $17,80 \%$ con reacciones $2+$ y $3+$ al CMT; pero son semejantes a los de Jiménez (2011) y a lo reportado por otros autores $(20,21,22)$.

De los 470 cuartos positivos al CMT fueron aislados 163 microorganismos involucrados en la patogénesis de la mastitis bovina durante el año de estudio. En la Tabla II se observa cómo S. aureus, con el $8,40 \%$, en promedio, como causa de mastitis subclínica, muestra un valor bajo comparado con otros países; por ejemplo, en el estado Zulia (Venezuela) se halló en el $17 \%$ de los casos (23), y en los países nórdicos el $S$. aureus fue aislado entre el 30 y el $40 \%$ de los casos subclínicos; en general, en los países industrializados el $S$ - aureus 
es el patógeno contagioso más aislado de casos de mastitis en vacas lecheras (24).

Los porcentajes de otros $S$. aureus coagulasa positivos (diferentes al S. aureus) fueron del $0,00 \%$. En otros estudios en Colombia, en el altiplano cundiboyacense, la prevalencia en un solo año fue de 4,04\% (25), valor superior al reportado en este trabajo, y en el Valle de Ubaté (26) encontraron el $0,15 \%$, mientras que en Venezuela fue del $23 \%$ (23).

Para el caso de S. agalactiae fue de 6,50\%, porcentaje que se considera muy bajo frente a lo reportado por Calderón y Rodríguez (25): 50\% en la misma zona en Colombia; por Contreras y Ordoñez (26): $35,40 \%$ en Colombia, y por Valero et al. (23): $26 \%$ en Venezuela. Países como Israel han erradicado este microorganismo con prácticas como el aislamiento en grupos de portadores, tratamientos con dosis de penicilinas y seguimiento bacteriológico (27). En Finlandia, Suecia y Noruega, el $S$. agalactiae es un germen poco común, con prevalencias menores al $1 \%$ (28), debido a procedimientos estrictos en los programas nacionales de control de la mastitis.

Tabla II. Resultados de los exámenes bacteriológicos en vacas con mastitis y su distribución proporcional, año 2013

\begin{tabular}{|l|c|c|}
\hline \multicolumn{2}{|c|}{ Número total de pruebas } & \multicolumn{2}{|c|}{643} \\
\hline Patógeno & 480 & $74,7 \%$ \\
\hline A. pyogenes & 3 & $0,5 \%$ \\
\hline S. agalactiae & 42 & $6,5 \%$ \\
\hline otros Streptococcus & 3 & $0,5 \%$ \\
\hline S. aureus & 54 & $8,4 \%$ \\
\hline Otros Staphylococcus CP & 0 & $0,0 \%$ \\
\hline Staphylococcus CN & 0 & $0,0 \%$ \\
\hline S. aureus + S. agalactiae & 3 & $0,5 \%$ \\
\hline levaduras & 1 & $0,2 \%$ \\
\hline Escherichia coli & 0 & $0,0 \%$ \\
\hline Corynebacterium bovis & 2 & $0,3 \%$ \\
\hline Acholeplasma spp & 9 & $1,4 \%$ \\
\hline Mycoplasma (No diferenciado) & 40 & $6,2 \%$ \\
\hline M. californicum & 3 & $0,5 \%$ \\
\hline M. alcalescens & 0 & $0,0 \%$ \\
\hline Otras mixtas & 3 & $0,5 \%$ \\
\hline CP: Coagulasa Positivo; CN: Co
\end{tabular}

El Corynebacterium bovis se aisló en el 0,30\% de 643 aislamientos; en el trabajo de Calderón y Rodríguez (25) fue aislado en el 8,44\%, valor muy superior a lo reportado por Gómez et al. (29), para la misma zona donde obtuvo el $2,50 \%$ y que sigue siendo un valor alto, para lo reportado por este estudio. Para diversos autores $(25,27,28), C$. bovis es un habitante normal del conducto del pezón, de donde se aísla sin estar asociado a mastitis aunque se asocia su aislamiento a un sellado deficiente de pezones, ya sea por mal manejo del producto o por la técnica empleada (23).

Estos estudios permiten afirmar que la mastitis por microorganismos contagiosos es la principal problemática en sistemas especializados en la producción de leche; mientras que los microorganismos de origen ambiental, como en el caso de los otros Streptococcus (S. uberis y $S$. dysgalactiae), representaron el $0,50 \%$. Es posible que estos patógenos estén disminuyendo debido a las buenas prácticas de prevención y control de la mastitis.

Las infecciones mixtas, $S$. aureus $+S$. agalactiae, representaron el $0,50 \%$; valores ligeramente superiores fueron reportados en Colombia por Contreras (26): el 1,90\%, y valores muy superiores fueron reportados por Andrade et al. (8) con el 8,14\%. En los resultados no se encontró crecimiento bacteriano en el medio de cultivo en el $74,70 \%$ de las muestras del presente estudio. En otros estudios no se obtuvieron crecimientos en el $15 \%$ (25) de las muestras que resultaron positivas a la mastitis subclínica mediante la prueba CMT. Las variaciones se pueden deber a la presencia de antibiótico o tratamientos que no fueron reportados en las vacas.

Se encontró $M$. californicum en el $0,50 \%$, y no se encontró $M$. bovis, que es en realidad el Micoplasma patógeno, que produce grandes pérdidas. En Colombia, si bien son frecuentes los brotes de mastitis clínicas y subclínicas resistentes a los tratamientos convencionales, no se había informado del aislamiento de Micoplasmas, por lo mismo, este es uno de los primeros reportes científicos de aislamiento de Mycoplasma californicum en Colombia, produciendo mastitis en vacas lecheras. M. alcalescens no se encontró en ninguna muestra. 
Roy José Andrade-Becerra, Zonia Elizabeth Caro-Carvajal, Martín Orlando Pulido-Medellín,

José Luis Porras-Vargas, Julio César Vargas-Abella

Los datos de estudios bacteriológicos previos fueron de suma importancia para sospechar de un brote de micoplasmosis (altos recuentos de celulas somáticas con bajos porcentajes de aislamiento de microorganismos patógenos), ya que coincide con los datos publicados por otros investigadores $(30,31)$. Otros elementos por tener en cuenta, y que también se hallan bien documentados (11, 31-32), fueron el alto número de animales con los cuatro cuartos comprometidos, la marcada baja de producción (aproximadamente 5 L/día), la falta de respuesta a las terapias antimicrobianas y, por último, la observación de un sedimento de aspecto arenoso en algunas de las muestras de leche positivas al aislamiento. Todos estos factores deberían ser tenidos en cuenta tanto por los veterinarios de campo como por los laboratorios de diagnóstico bacteriológico, a fin de detectar brotes de esta enfermedad. Si bien los porcentajes de aislamiento de Micoplasmas son bajos a nivel mundial, las pérdidas económicas que ocasionan son considerables, debido a la ineficacia de la antibioterapia, a la rápida diseminación de la enfermedad, a la alta pérdida de la producción lechera y a los inconvenientes de manejo, como aislamiento de animales, ordeñar por separado y no ordeñar con la misma máquina todos los animales. Por tales motivos, en los últimos años se hace énfasis en el diagnóstico de Micoplasmas como causa de mastitis. En Colombia los productores lecheros se preocupan cada vez más en mejorar su producción con la incorporación de nuevas tecnologías y el control sanitario.

El caso de infección mixta donde se encuentra micoplasma involucrado se contó como mastitis por Mycoplasma spp. Con las infecciones que se mezclan de $S$. agalactiae con $S$.aureus o con otros gérmenes solo se reportó el que mostraba mayores conteos.

\section{CONCLUSIONES}

Durante el año de estudio se encontró un $3.14 \%$ de cuartos positivos al CMT, con grados superiores a $2+$. Streptococcus agalactiae se aisló en el 9,73\% de los cuatros, y se convirtió en el principal patógeno aislado. Staphylococcus aureus fue aislado, en promedio, en el 6,00\%. Corynebacterium bovis se aisló en el 0,30 \% de los casos. E. coli no se aisló. Mycoplasma bovis no se encontró en ninguna muestra. M. californicum se recuperó en el 0,50\%, Levaduras en el 0,20\% y Acholeplasma spp. Se aisló en el 0,40\%.

A partir de los resultados obtenidos en este estudio se considera de suma importancia tener en cuenta los Micoplasmas como agentes productores de mastitis en fincas lecheras del altiplano Boyacense.

\section{REFERENCIAS}

(1) Murphy JM. Mastitis the struggle for understanding. J. Dairy Sci 2006; 39: 1768-1773.

(2) Rainard PPB. Dinamics of nonclinical bovine intramammary infections with major and minor pathogens. Am. J. Vet Res 2002; (43): 2143- 2146.

(3) David GP, Jackson G. The collection and interpretation of herd mastitis data. Br Vet J 2004; (140): 107-114.

(4) Philpot WN. Modern mastitis management. Vet. Scope $2011 ;$ 10(1): 2-11.

(5) David GP, Jackson GD. Mastitis losses. J Am Vet Med Assoc 2012; 190(20): 1129-1132.

(6) Gill RS, Howard WH, Leslie KE. Economics of mastitis control in Ontario dairy herds. Am J Vet Res 2011; (43): 512-523.

(7) Philpot WN, Pankey JW. Hygiene in the prevention of udder infections III. Effectiveness of 59 teats dips for reducing bacterial populations on the skin. J Dairy Sci 2010; (68): 421-428.

(8) Andrade R, Pulido M, Rodríguez C, Sanidad de ubre, Calidad de leche. Tunja, Colombia: Uptc. 170 p. 2012.

(9) Kruze J. Etiología y epidemiología de la mastitis. Rev Holstein Chile 2002; (34): 14-15.

(10) León B. Frecuencia de aislamiento de los principales agentes de mastitis en el sur de Chile. Rev Cooprinforma 2007; (40): 1-6.

(11)Zurita E. Bovine mastitis caused by a Mycoplasma species. Cornell Vet 2012; (52): 582-591.

(12)Martin S, Meek A. Epidemiología veterinaria. Principios y métodos. Zaragoza, España: Editorial Acribia. 2009.

(13) IDEAM. Instituto de Hidrología Meteorología y Estudios Ambientales de Colombia. (en línea) 
2012 (fecha de acceso el 13/02/2014). Disponible en: http://www.ideam.gov.co/jsp/index.jsf

(14) National Mastitis Council (NMC). Microbiological Procedures for the Diagnosis of Bovine Udder Infection and Determination of Milk Quality. 4 th ed. Verona: USA. 2009.

(15)Freundt E. Culture media for classic Mycoplasma. J Dairy Research 2003; (24): 127-135.

(16)Tully J. Test for digitonin sensitivity and sterol requirement. J. Anim. Prod 2012; (1): 355-362.

(17) Razin S, Cirillo V. Sugar fermentation. Methods in Mycoplasmology 2003; 1: 337-343. (18) Alonso FR, Maldonado C, Castillo A, Perfiles de incidencia de Mastitis Bovina. Vet Ven 2010; (XLVII): 11- 29.

(19)Jiménez MR. Estudio preliminar de la mastitis subclinica en vacas productoras de ciertas zonas altas del estado de Mérida. Rev Col Cienc Pec 2011; (IV): 82-89.

(20) Cole EJ, Painter EV, Schnepper GH. Detection Eficiency of Mastitis Screening Test. J Milk Food Technol 2005; (28): 5-8.

(21)Fustes E, Ávila C, Ortega L. Mastitis bovina: efecto sobre la producción lechera y la economía agropecuaria en Cuba. Rev Salud Anim 2005; (7): 91-100.

(22) Wesen DP, Luedecke LO, Forster TL. Relationship between California Mastitis Test Reaction and Bacteriological Analyses of stripping samples. J. Dairy Sci 2008; (61): 620-626.

(23) Valero K, Valbuena E, Chacon F, Olivares Y, Castro G, Briñez W. Patógenos contagiosos y ambientales aislados de cuartos mamarios con mastitis subclínica de alto riesgo en tres fincas del estado de Zulia. Rev Cientif FCV-LUZ 2010; XX (5): 498-505.
(24)Osteras O, Martin S. Possible risk factors associated with penicillin-resistant strains of Staphylococcus aureus from bovina subclinical mastitis nearly lactation. J Dairy Sci 2009; (2): 345-356.

(25)Calderón A, Rodriguez V. Prevalencia de mastitis bovina y su etiología infecciosa en sistemas especializados en producción de leche en el altiplano Cundiboyacense (Colombia). Rev Col Cien Pec 2008; (21):582-589.

(26)Contreras D, Ordoñez P. Prevalencia y dinámica de la mastitis bovina en el valle de Ubaté. Rev Col Cienc Pec 2004; (14): 10-16.

(27) Sargeant J, Leslie K, Shirle J, Pulkrabek B, Lim G. Sensitivity and specificity of somatic cell count and California mastitis test for identifying intramammary infection in early lactation. J Dairy Sci 2010; (48): 1212-1423.

(28) Rebhum W, Guard C, Richard C. Relationship between lactation measures of somatic cell concentration. Disease of dairy cattle 2006; (1): 122-136.

(29) Gómez L, Pinilla R, Jaramillo E. Mastitis bovina y su etiología infecciosa en la región de Úmbita (Boyacá). Rev Colom Cienc Pecua 2009; 18(2): 16-20.

(30) Bushnell RB. Mycoplasma mastitis. Vet Clin North Am (Large Anim. Pract) 2004; (6): 301-312.

(31)Gonzalez R, Sears P. Diagnosis, control, and effect on milk production of Mycoplasma bovis intramammary infections. J Dairy Research 2003; (26): 681-684.

(32)Boughton E. Mycoplasma bovis mastitis. Vet Bull 2009; (49): 377- 388. 